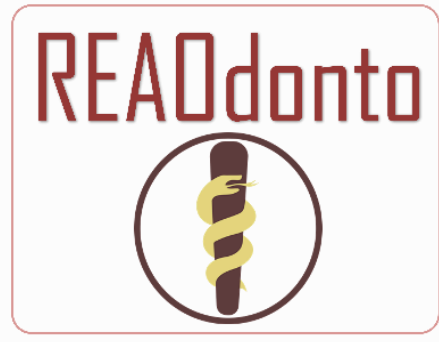

Revista Eletrônica Acervo Odontológico

\section{REVISÃO BIBLIOGRÁFICA}

Recebido em: 6/2020

Aceito em: $7 / 2020$

Publicado em: 10/2020

\title{
Desafios da educação em saúde bucal na adolescência
}

\author{
Defiances of oral health education in adolescence
}

\author{
Deficiencias de la educación en salud oral en la adolescencia
}

Maria das Graças Silva Sarmento ${ }^{1 *}$, Odivaldo Alves dos Santos ${ }^{2}$, Marly Monteiro Lima ${ }^{3}$.

\begin{abstract}
Resumo: Descrever os desafios da educação em saúde bucal na adolescência. A fase da adolescência é considerada muito crítica para a saúde bucal, pois é marcada por conflitos, comportamentos e transformações decorrentes do período de transição entre a infância até a fase adulta. Foi realizado um levantamento bibliográfico selecionando revistas indexadas disponíveis nas bases de dados online LILACS e SCIELO utilizando os descritores "Odontologia"; "Adolescente", "Educação", "Saúde Bucal", que compreendiam o período de 2015 a 2020. Dos 17 artigos evidenciou-se falta de cuidado direcionado ao adolescente, apesar dos vários avanços já alcançados, e a necessidade de ações mais eficientes que melhorem o acesso ao conhecimento e o reforço da autoestima do adolescente, indicando importância que o Cirurgião-Dentista tem de proporcionar um atendimento integral nesta fase. O Cirurgião-Dentista deve estar atento para as mudanças hormonais que esta fase exige, ser sensível aos fatores socioeconômicos envolvidos que afeta a saúde oral do indivíduo promovendo um atendimento eficaz.
\end{abstract}

Palavras-chave: Odontologia, Adolescente, Educação em saúde bucal.

\begin{abstract}
To describe the challenges of oral health education in adolescence. The adolescence phase is considered to be very critical for oral health, as it is marked by conflicts, behaviors and changes resulting from the transition period from childhood to adulthood. A bibliographic survey was carried out, selecting indexed journals available in the online databases LILACS and SCIELO using the descriptors "Dentistry"; "Adolescent", "Education", "Oral Health", which comprised the period from 2015 to 2020. Of the 17 articles, there was a lack of care directed at adolescents, despite the various advances already achieved, and the need for more efficient actions that improve access to knowledge and reinforce the adolescent's self-esteem, indicating the importance that the Dental Surgeon has to provide comprehensive care at this stage. The Dental Surgeon must be aware of the hormonal changes that this phase requires, be sensitive to the socioeconomic factors involved that affect the oral health of the individual, promoting effective care.
\end{abstract}

Keywords: Dentistry, Adolescent, Health Education Dental.

Resumen: Describir los desafíos de la educación en salud bucal en la adolescencia. La etapa de la adolescencia se considera muy crítica para la salud bucal, ya que está marcada por conflictos, conductas y

\footnotetext{
1 Instituto Leônidas e Maria Deane (ILMD/FIOCRUZ). Manaus - AM. *E-mail: mgsarmento@hotmail.com

2 Faculdade Master de Paraupebas (FAMAP). Parauapebas - PA.

3 Instituo de Ensino Superior de Fortalez (IESF). Fortaleza - CE.
} 
transformaciones resultantes del período de transición de la niñez a la edad adulta. Se realizó una encuesta bibliográfica, seleccionando revistas indexadas disponibles en las bases de datos en línea LILACS y SCIELO utilizando los descriptores "Odontología"; "Adolescente", "Educación", "Salud Bucal", que comprendió el período de 2015 a 2020. De los 17 artículos, hubo una falta de atención dirigida a los adolescentes, a pesar de los diversos avances ya logrados, y la necesidad de acciones más eficientes que mejorar el acceso al conocimiento y el fortalecimiento de la autoestima de los adolescentes, indicando la importancia que tiene el Cirujano Dentista de brindar una atención integral en esta etapa. El Cirujano Dentista debe ser consciente de los cambios hormonales que requiere esta fase, ser sensible a los factores socioeconómicos involucrados que afectan la salud bucal del individuo, promoviendo una atención eficaz.

Palabras clave: Odontología, Adolescente, Educación en salud dental.

\section{INTRODUÇÃO}

O corpo humano passa por períodos de grandes mudanças no decorrer da vida, tanto biológicas, como morfológicas e fisiológicas. A fase da adolescência (entre 15 a 19 anos) é considerada muito crítica para a saúde bucal, pois é marcada por conflitos, comportamentos e transformações decorrentes do período de transição entre a infância até a fase adulta, evidenciando assim os maus hábitos alimentares e a higiene bucal. Diante de tantas descobertas e dilemas, o adolescente deve ser instruído com relação ao seu modo de pensar, agir, suas emoções, aparência, autoestima e tudo que possa interferir no seu bem-estar, e a saúde bucal está diretamente ligada ao bem-estar do indivíduo (ARAÚJO MVA, et al., 2017; LIMA HT, et al., 2016; GONÇALVES LFF, et al., 2016).

Ainda atualmente, existem jovens que não tem acesso à saúde, seja por questões financeiras ou falta de motivação. A prevalência de cárie e doenças periodontais nesta época se dá devido a dieta rica em alimentos cariogênicos e às mudanças hormonais que alteram a microbiota bucal (ARAÚJO MVA, et al., 2017). Estudos apontam que a cárie dentária é a patologia que mais acomete a cavidade bucal nesta faixa etária, e a ingestão de alimentos ricos em açúcar é um hábito comum nesta fase da vida (LIMA HT, et al., 2016).

Dentre esses alimentos podemos destacar as balas, salgadinhos, refrigerantes, biscoitos e chocolates. É um cardápio variado em que os jovens têm fácil acesso na própria escola, e muitas vezes em casa sem a supervisão dos pais. Além da dieta, fatores socioeconômicos por sua vez também contribuem para uma saúde bucal precária, jovens de classe média baixa possuem maior índice de cárie por não terem acesso a tratamentos odontológicos (RODRIGUES MA e PEREIRA PF, 2018). A criação de políticas públicas voltadas para a saúde bucal dos adolescentes diminuiria os impactos na qualidade de vida e o ideal é que fossem implementadas em todas as escolas (FILGUEIRA ACG, et al., 2016).

A cárie é uma doença multifatorial, transmissível, que progride de acordo com a falta de higienização dos dentes, acarretando na desmineralização dentária (SILVA JUNIOR IFS, et al., 2018). A perda de elementos dentários precoce devido ao acometimento por cárie influencia diretamente na autoestima do jovem, interferindo nas atividades diárias, aparência e fala. Alterações mastigatórias, anormalidades de oclusão e apinhamento dentário são algumas das consequências que mais ocorrem (LUNARDELLI SE, et al., 2016).

Nesta fase, através do levantamento feito pelo Projeto SB Brasil 2010 foi verificado uma prevalência de cárie de $56 \%$ entre os jovens. Com esses dados é possível implementar no SUS (Sistema Único de Saúde) novas políticas públicas visando a prevenção e promoção da saúde bucal para os adolescentes. Muitos deles não recebem a atenção necessária dos pais, como antes na infância, e acabam mais suscetíveis à cárie e as doenças periodontais (OLIVEIRA AN, et al., 2011; LIMA HT, et al., 2016; VASCONCELOS FGG, et al., 2018).

$\mathrm{E}$ as doenças periodontais que podem acometer a cavidade oral dos jovens com mais frequência são a gengivite, que é uma inflamação do tecido gengival podendo haver ou não sangramento espontâneo, e a periodontite, que é a progressão da gengivite e que se trata de uma inflamação mais grave onde ocorre a

REAOdonto | Vol. 2 | e4249 | DOI: https://doi.org/10.25248/REAOdonto.e4249.2020 Página 2 de 6 
reabsorção óssea ao redor do elemento dentário. Ambas são causadas pelo acúmulo de bactérias que causam a inflamação dos tecidos. O diagnóstico é feito pelo cirurgião-dentista através de avaliação clínica e exames de sondagem gengival para avaliar o grau da inflamação (SPEZZIA S, 2018).

Diante desta realidade o profissional da área da saúde juntamente com a escola tem um papel fundamental para a reversão desse quadro, buscando criar ações educativas para que o jovem compreenda a importância de cuidar da saúde bucal e de que maneira isso pode afetar do seu dia a dia de forma positiva. Os jovens devem receber palestras motivacionais, aprendendo técnicas corretas de escovação, orientações sobre a higienização, e os riscos causados pela cárie e as doenças periodontais (SPEZZIA S, 2018).

A importância de uma equipe multidisciplinar desempenhar ações fundamentais de cuidados com a saúde bucal frente a pacientes jovens, atuando de forma dinâmica e decisiva pode evitar possíveis agravos. Cabe ao cirurgião-dentista priorizar o atendimento e dar uma ênfase especial em atividades preventivas, sendo eficaz na abordagem com o adolescente, uma vez que o tratamento preventivo irá diminuir consideravelmente os índices de cárie e doenças periodontais nesses pacientes.

O objetivo deste trabalho é justamente relatar os desafios enfrentados pelos os profissionais de odontologia na educação em saúde bucal entre os adolescentes.

\section{REVISÃO BIBLIOGRÁFICA}

Trata-se de um levantamento bibliográfico que consistiu em organizar, esclarecer e resumir as principais obras existentes, bem como fornecer citações completas abrangendo o espectro de literatura relevante em uma área. Foram incluídos artigos científicos de revistas indexadas disponíveis nas bases de dados online como Scientific Electronic Library Online (SCIELO) e Literatura Latino-Americana em Ciências da Saúde (LILACS). Utilizando os descritores "Odontologia"; "Adolescente", "Educação", "Saúde Bucal", estudos publicados no Brasil, em idioma português e que compreendiam o período de 2015 a 2020.

Os artigos selecionados foram submetidos a uma leitura rigorosa do texto completo e fichados para identificar os assuntos relacionados a educação bucal e adolescente, analisando os artigos científicos de acordo com os seguintes aspectos: título, autor, ano, procedência/periódico, principais resultados e conclusões.

Com a organização dos dados foi possível analisar e identificar os principais resultados dos artigos selecionados e com isso descrever como deve ser realizada a abordagem do cirurgião-dentista com o adolescente para promover ações preventivas na odontologia. A análise se deu através da triangulação dos dados coletados, com a análise crítica do autor da pesquisa confrontados com a literatura.

A adolescência é considerada um período de risco para doenças bucais. Os adolescentes não são beneficiados pelos os cuidados e atenções dispensadas às crianças e nem desfrutam de um atendimento direcionado a eles como aos adultos, portanto há a necessidade de compreender a individualidade desses pacientes, procurando atendê-los de forma adequada para que a meta de prevenção seja alcançada (OLIVEIRA AN, et al., 2011). Constituem $20 \%$ da população mundial, com cerca de 34 milhões no Brasil; esta fase é caracterizada por insegurança com a vida e contradições de comportamento. Há muitas alterações biológicas e associada a situações adversas como pobreza, desigualdades sociais e o estilo de vida que o indivíduo carrega torna essa fase muito complexa e de muitos riscos (ARAÚJJO MVA, et al., 2017, BRASIL, 2008).

No Brasil, há escassez de literatura disponível abordando a saúde bucal dos adolescentes e o seu perfil periodontal, sendo necessária a realização de mais estudos com intuito de demostrar a situação da saúde bucal desse grupo (BARBOSA ALL, et.al, 2019).

Apesar da relação direta entre aumento da idade e aumento do CPO-D (dentes permanentes cariados, perdidos e obturados), os dados do Levantamento Epidemiológico (2010), apontam para uma redução de $30 \%$ do CPO-D de adolescentes entre 15 e 19 anos. Condições sóciodemográficas, psicossociais e o estilo 
de vida determinam os comportamentos do adolescente frente aos hábitos de higiene e à própria saúde (LIMA HT, et al., 2016; VASCONCELOS FGG, et al., 2018; SILVA JUNIOR IFS, et al., 2016).

Nessa fase o jovem pode adquirir conhecimentos para reforçar atitudes e comportamentos positivos, que serão mantidos futuramente. Hábitos inerentes à faixa etária pesquisada, como por exemplo, sexuais e compartilhamento alimentar exigem atenção dos programas educativos que visem a promoção de saúde. É fundamental que o cirurgião-dentista, além de fornecer orientações sobre higiene bucal, também, dissemine informações que ampliem a visão das pessoas para além dos aspectos de higiene bucal focados na cárie dental (MARÍN C, et.al, 2016; SPEZZIA S, 2018).

A alta frequência de ingestão de alimentos e bebidas açucaradas na população adolescente atualmente é preocupante e a relação do nível socioeconômico com a saúde bucal, pois estas condições exercem influência sobre o comportamento e o autocuidado, assim como na autopercepção da saúde bucal. Apesar desta relação, Rodrigues MA e Pereira PF (2018), não observaram associação entre o CPOD (dentes permanentes cariados, perdidos e obturados) e o nível socioeconômico dos adolescentes classificado pelo critério de posse de bens e acesso a serviços. Resultados semelhantes foram encontrados em estudo realizado com alunos de duas escolas públicas localizadas na região sul do país.

O que se pode observar é que não há uma homogeneidade dentro do país com relação aos dados sobre os adolescentes. E que o acesso odontológico, as patologias relacionadas que este grupo apresenta e a percepção de saúde bucal que este grupo possui se modifica de acordo com cada região. Em um arquipélago do Estado do Pará, Araújo MVA, et al. (2017), encontraram um índice CPOD (dentes permanentes cariados, perdidos e obturados) de 187 adolescentes e concluiu que quanto maior o índice maior o relato de dor, e somente $33,68 \%$ estavam satisfeitos com sua saúde bucal.

Em comunidades do Pará, localizadas as margens do rio Maró, Amaral RC, et al. (2017) examinaram 416 crianças e encontrou o índice ceod (dentes decíduos cariados, com extração indicada, perdidos devido à cárie ou obturados) médio de 5,3 $( \pm 4,5)$ e o índice CPOD (dentes permanentes cariados, perdidos e obturados) de 4,0 ( $\pm 3,1)$, com predominância do cariado. Esses dados muitos elevados em relação ao nível nacional indicando a necessidade de acesso a atendimentos odontológicos e de políticas de promoção de saúde.

Em faculdade pública na cidade de Manaus/AM, em uma análise retrospectiva do tipo transversal dos anos de 2017 e 2018, 60\% apresentaram presença de sangramento, 68,3\% dos pacientes adolescentes apresentaram presença de gengivite e $13,3 \%$ com periodontite o que demonstra a alta prevalência de gengivite, indicado a necessidade uma abordagem mais contundente com esta população. Ao analisar um Instituto Federal de Educação, Ciência e Tecnologia em 215 adolescentes, o cálculo dentário foi a periodontopatia mais severa e $31,5 \%$ demonstrou necessidade de tratamentos ortodôntico (BARBOSA ALL, et al., 2019; FILGUEIRA ACG, et al., 2016).

Em um estudo transversal com 409 adolescentes em 3 municípios do Sul do Brasil, pesquisadores avaliaram a relação da cárie e autoestima através da Escala de Rosenberg e concluíram que os aspectos de autoestima foram significativamente associados com a presença de dentes cariados, isto é quanto maior a quantidade de dentes cariados pior era a autoestima dos adolescentes, o que indica que na saúde pública esse público deve ter uma atenção especial principalmente no fator psicológico (LUDARNELLI SE, et al., 2016). Noutro estudo descritivo alunos de uma escola de rede pública de um município de Santa Catarina, 144 adolescentes foram pesquisados para se conhecer a percepção e o nível de informação dos adolescentes sobre saúde bucal. Os adolescentes demonstraram que já tiveram acesso a informações sobre saúde bucal, cuja fonte era o próprio dentista e que receberam atendimento odontológico ao menos 1 vez ao ano, o que se concluiu que eles tiveram acesso ao conhecimento sobre saúde bucal e possuíam uma boa autopercepção sobre o assunto (MARÍN C, et al., 2016).

Um estudo de associação do estado nutricional, fatores socioeconômicos e comportamentais com a saúde bucal de adolescentes de 15 a 19 anos em escolas públicas do município de Viçosa - MG, verificouse que o excesso de peso dos adolescentes configura uma pior saúde bucal, possivelmente devido ao 
consumo de alimentos cariogênicos, o que implica no estabelecimento de ações mais especificas direcionadas a esse quadro (RODRIGUES MA e PEREIRA PF, 2018).

Souza RLL, et al. (2017), avaliaram a relação entre a necessidade de tratamento odontológico devido à cárie dental e níveis de ansiedade em adolescentes através de um estudo de corte transversal com 89 adolescentes entre 11 e 19 anos de idade. A necessidade de tratamento odontológico devido a cárie foi muito maior entre os adolescentes com maiores níveis de ansiedade, em especial os do sexo feminino, demonstrando a necessidade de um cuidado especial não só apenas com a fase assim como com o sexo do adolescente.

Spezzia S (2018), já havia averiguado as modificações hormonais que as meninas apresentam na adolescência, visto que o sexo masculino não possui uma alteração tão marcante. A doença periodontal recebe influência da ação dos hormônios sexuais. Estudantes das escolas privadas possuem uma melhor saúde oral do que de escolas publica, possivelmente por causa do acesso aqueles têm a água tratada, saúde e educação. É notório que os meninos são detentores de hábitos de higiene bucal ruins se comparados com as meninas. Há diferença significativa nos serviços odontológicos entre as regiões do país: a melhor situação foi encontrada no Sul, e as piores no Norte e Nordeste do Brasil. Mas é óbvio que os adolescentes necessitam e carecem de atendimento odontológico público, de políticas de intervenção na escola no processo saúde-doença (SILVA JUNIOR IFS, et al., 2016).

A condição socioeconômica é um dos determinantes sociais mais importantes na Odontologia. Adolescentes com melhores condições econômicas apresentam menor percentual de ansiedade. Em estudo nacional o número de desassistidos é muito maior entre os mais pobres, e esses são os que também tem maior dificuldade de conseguir atendimento (SOUZA RLL, et al., 2017). Para desenvolver uma prática de educação em saúde mais efeito com ênfase no autocuidado, Borges MIMP, et al. (2019), iniciaram um estudo fundamentado nas Metodologias Ativas de Ensino-Aprendizagem, por meio de duas oficinas, com 40 adolescentes. Houve uma participação intensa dos adolescentes dentro das oficinas, e revelou-se exitosa, pois os adolescentes puderam compartilhar suas adversidades familiares e cotidianas e debater sore o autocuidado na saúde bucal.

Gonçalves LFF, et al. (2016), realizaram um relato de experiência com um grupo de adolescentes (idade entre 11 e 16 anos), sexo feminino em escolas públicas em Caicó-RN. Nas experiências envolvendo palestras e oficinas lúdicas, os autores conseguiram desempenhar a função social e verificar que o público vive em vulnerabilidade social somado ao total descaso das políticas públicas.

A educação lúdica pode ser utilizada como recurso para produzir conhecimento crítico e despertar a reflexão sobre os hábitos e conduzir conceitos de prevenção e promoção em saúde. Aguiar NL, et al. (2018), desenvolveram com alunos do curso de Odontologia da Universidade Federal do Pará um jogo de cartas intitulado "Jogo SB", com temas sobre saúde bucal. Participaram da pesquisa 29 estudantes, (51,7\%) do sexo feminino (48,3\%) do sexo masculino, com idades entre 11 a 17 anos. A adesão ao jogo foi imediata, o que demonstrou que técnicas alternativas de educação em saúde torna a aquisição desses conhecimentos algo dinâmico.

Os adolescentes demonstraram espontaneidade, interesse e motivação atividades lúdicas que se relacionem a temas como sexualidade e sexo oral, demonstrando que há o despojamento, na abordagem do tema e nenhum constrangimento o que reforça a necessidade de os profissionais estarem difundindo conhecimentos de saúde bucal neste público. (BORGES MIMP, et al., 2019). Uma das estratégias para atuar com os adolescentes é desenvolver maneiras para que o diálogo obtenha uma discussão e reflexão de comportamento, abolindo a unidirecional, dogmática e autoritária, com a meta de apenas informar. Cabe aos profissionais de saúde inseridos nos serviços de saúde captar o universo do adolescente e reforçar ações de educação em saúde (FILGUEIRA ACG, et al., 2016).

Portanto aspectos da autoestima estão associados fortemente com a presença de dentes cariados, e medidas de intervenção direcionadas à melhoria das condições de saúde oral pode contribuir para a construção e manutenção de um alto nível de autoestima no adolescente (LUNARDELLI SE, et al., 2016).

REAOdonto | Vol. 2 | e4249 | DOI: https://doi.org/10.25248/REAOdonto.e4249.2020 Página 5 de 6 
Para enfrentar os desafios dessa fase destaca-se a importância do trabalho com o elemento lúdico, proporcionado por atividades como brincadeiras e jogos. Nesse aspecto, a ludicidade não pode ser vista apenas como diversão, uma vez que facilita a aprendizagem e o desenvolvimento pessoal, social e cultural, possibilitando ao ser humano aprender de modo descontraído melhorando até a saúde mental facilitando os processos de socialização, comunicação e expressão construindo o conhecimento (GONÇALVES LFF, et al., 2016).

\section{CONSIDERAÇÕES FINAIS}

O Brasil evoluiu muito em relação a sua saúde bucal tanto nas crianças como com os adolescentes. Mesmo com a melhora dos índices odontológicos, há a necessidade de ampliação dos serviços ofertados e um olhar mais cuidadoso do profissional em odontologia para as minúcias que a puberdade exige, já que é uma fase de mudança em que o indivíduo modifica seu modo de ver o mundo e a si próprio. O CirurgiãoDentista deve estar atento para as mudanças hormonais e psicológicas que esta fase exige, ser sensível aos fatores socioeconômicos envolvidos que afeta a saúde oral do indivíduo, tornando o aprendizado ao adolescente acessível e lúdico fortalecendo sua autoestima e sedimentando conhecimentos que serão o suporte para uma vida saudável em todos os aspectos. Foi identificado por este estudo uma escassez na literatura sobre o tema, falta de ações de promoção de saúde para esta faixa etária, uma falta de homogeneidade de dados sobre os adolescentes, uma diferença entre os serviços odontológicos nas regiões do país, e como as diferenças econômicas e de gênero afeta a prevalência de doenças orais na adolescência. É necessário que o profissional esteja capacitado e que haja uma ampliação de serviços e fortalecimentos de políticas públicas para o acesso odontológico aos mais desprovidos.

\section{REFERÊNCIAS}

1. AGUIAR NL, et al. Jogo SB: Estratégia lúdica de educação em saúde bucal para adolescentes na Amazônia. Interdisciplinary Journal of Health Educations, 2018; 3:1-2.

2. AMARAL RC, et al. A relação entre a saúde bucal e a cárie dentária em oito comunidades ribeirinhas - PA, Brasil. Rev. Bras. Odontol., Rio de Janeiro, jan./mar, 2017; 74(1):18-22.

3. ARAÚJO MVA, et al. Prevalência de cárie dentária, autopercepção e impactos em saúde bucal em adolescentes na ilha do Marajó - PA. RDAPO: Revista Digital de Academia Paraense de Odontologia, Belém, 2017; 19(1):11-17.

4. BARBOSA ALL, et al. Perfil periodontal dos pacientes adolescentes atendidos na policlínica odontológica da UEA. Revista de Ciências da Saúde da Amazônia, Manaus, 2019; 1: 25-37.

5. BRASIL. Ministério da Saúde. Saúde do Adolescente: competências e habilidades. Brasília: Ministério da Saúde; 2008. http://bvsms.saude.gov.br/bvs/publicacoes/saude_adolescente_competencias_habilidades.pdf.

6. BORGES MIMP, et al. Educação em saúde no segmento adolescente sob a perspectiva das metodologias ativas de ensino-aprendizagem. Revista da ABENO, 2019; 19 (3): 37-49.

7. FILGUEIRA ACG, et al. Saúde Bucal de Adolescentes Escolares. Holos, 2016; 1(32):162-172.

8. GONÇALVES LFF, et al. M. Promoção de saúde com adolescentes em ambiente escolar: relato de experiência. SANARE-Revista de Políticas Públicas, jun./dez. 2016; 15 (2): 160-167.

9. LIMA HT, et al. A prevalência de cárie dentária em adolescentes de 12 anos no Brasil. Jornada Odontológica dos Acadêmicos da Católica - JOAC, 2016; 2(2).

10. LUNARDELLI SE, et al. Autoestima e cárie dentária em Adolescentes: um estudo seccional. Rev Odontol. UNESP, Araraquara, nov./dez., 2016; 45(6). http://dx.doi.org/10.1590/1807-2577.08116

11. MARÍN C, et al. Percepção e Informação sobre saúde bucal: estudo com adolescentes em uma escola pública. Revista Saúde e Pesquisa, set./dez., 2016; 9(3):499-506.

12. OLIVEIRA AN, et al. Promoção de saúde bucal para adolescentes. Arq Odontol, Belo Horizonte, dez., 2011; 47(2):64-67.

13. RODRIGUES MA, PEREIRA PF. Relação da cárie com estado nutricional, fatores sociais e comportamentais em adolescentes de 15 a 19 anos. RASBRAN - Revista da Associação Brasileira de Nutrição, São Paulo, jul./dez., 2018; 9(2):103-110.

14. SILVA JÚNIOR IFS, et al. Saúde Bucal do Adolescentes: Revisão de Literatura. Adolesc. Saude, Rio de Janeiro, ago., 2016; 13(1):95-103.

15. SOUZA RLL, et al. Necessidade de Tratamento Odontológico e níveis de ansiedade em adolescentes. Revista Bahiana de Odontologia, jun, 2017; 8(2):37-42.

16. SPEZZIA S. Alterações Periodontais na Adolescência. Braz J Periodontol, mar., 2018; 24(1):43-47. VASCONCELOS FGG, et al. Evolução dos índices CEO-D/CPO-D e de Cuidados Odontológicos em Crianças e Adolescentes com Base no SB Brasil 2003 e SB Brasil 2010. Revista Brasileira de Ciências da Saúde, 2018; 24(4):333-340.

REAOdonto | Vol. 2 | e4249 | DOI: https://doi.org/10.25248/REAOdonto.e4249.2020 Página 6 de 6 